\title{
MOVEMENT RECEPTORS IN DECAPOD CRUSTACEA
}

\author{
By C. A. G. WIERSMA ${ }^{1}$ \\ California Institute of Technology. \\ From the Plymouth Laboratory and Department of Zoology, Cambridge
}

(With Text-figures I-3)

The sense organ of Carcinus maenas located in the joint between the dactyloand propodite possesses several types of sense cells (Wiersma \& Boettiger, I958). The most interesting types react to unidirectional displacement of the dactylopodite with equal sensitivity over the total possible arc of movement. Velocity has little influence on the discharges of the movement fibres since the maximum rate of firing is reached at a speed which is relatively little faster than the threshold. Fibres with marked differences in threshold are present for both extension and flexion. Other units in the organ react like conventional stretch receptors and give continuous discharges as long as the joint is near one of the extreme positions. Again different units are present for extension and for flexion. These position fibres are on the whole smaller than the movement fibres and their cell bodies have a more peripheral location in the organ.

Organs similar to this PD-organ of Carcinus are known to be present in other joints and in other species (Alexandrowicz, 1958, and personal communication). The purpose of the present investigation was to determine their functions and particularly to find out whether they possess movement receptors.

\section{METHODS AND MATERIAL}

Unit analysis of fibres reacting to manipulation of specific joints was made by preparing single fibres or small bundles according to the methods described elsewhere (Wiersma \& Boettiger, 1958). In this investigation all fibres were prepared from the nerve or nerves in the meropodite. As it was not the purpose to study the relation between speed of movement and the discharge rate in the movement fibres, but merely to demonstrate their presence or absence, manual manipulation of the joints sufficed. For registration purposes a simple mechanical device was used which correlated the movements of the carpo-dactylopodite joint with the displacement of the second beam of a Cossor oscilloscope. For this purpose the knob controlling the vertical position of this beam was replaced by a brass pulley over which a thread without end

\footnotetext{
1 John Simon Guggenheim Memorial Fellow.
} 
was looped twice. The lower thread was similarly looped over the propodite at a convenient distance from the joint and continued to a pulley located on the other side of the preparation and then back to the brass pulley. By adjusting the tension in the thread and the place of the loop around the propodite, it was possible to establish a satisfactory relation between the vertical displacement of the beam and the maximum arc of movement of the joint. Since the brass knob was moved by hand, the displacement was not smooth, with resulting irregularities in the discharges, especially from the most sensitive movement receptors. But the method permitted clear distinction between the properties of most fibres.

In many preparations the nerve fibre bundles from which proprioceptive responses were obtained were subsequently traced to the organs from which they originated. To this end staining with methylene blue and additional exposure of the nerve bundles and organs were used.

The animals investigated were Carcinus maenas, Maia squinado, Eupagurus bernhardus, Homarus vulgaris and Palinurus vulgaris. The experiments on the latter four species were performed at Plymouth. In each animal the organs of the walking legs were investigated. Sea water was used as a physiological solution. For Maia a special solution was also tried, but no noticeable difference was observed, possibly because each experiment continued for at most two hours.

\section{RESULTS}

\section{The PD-organs of various species}

A comparison of the responses from the PD-organs of the other species with those of Carcinus, described elsewhere (Wiersma \& Boettiger, I958), shows that they are greatly similar. In all cases a small bundle of nerve fibres can be isolated in the meropodite, containing axons responding to different manipulations of the joint. In Palinurus, especially, fibres in another bundle also respond. It could be shown that these responses do not originate in the organ but from sense cells of hairs located on the distal part of the propodite, which are bent by the dactylopodite when it nears the extreme flexed position. These hairs form only part of the cluster present and on touch the signals in their fibres do not differ from those which are not stimulated when the dactylopodite is bent. It seems therefore unlikely that they have a proprioceptive function.

The four main types of fibres, opening and closing movement fibres, opened and closed position fibres, were regularly obtained from the PD organs of all genera with the exception of Homarus. In the PD nerve bundle of the latter closed position fibres were usually absent. Since all but the fourth walking legs are either chelated or subchelated in this species, it seemed possible that the stop provided by the propodite might be responsible. Therefore the fourth leg, in which the dactylopodite can move through as 
large an arc as in other genera, was especially used, but tonically firing closed position fibres were here as rare as in the other legs.

For a few preparations from different species, a count was made of the number and type of fibre which could be prepared from one PD nerve bundle. Because of damage such counts will not show the actual number of active units, but they will provide an approximation of the numerical relation of the different fibre types. In Table $I$ the results are shown.

TABLE 1. NUMBERS OF REACTING AXONS PREPARED IN THE PD-NERVE BUNDLES OF VARIOUS SPECIES

$\begin{array}{lccccc}\text { Carcinus } & \text { Opener } & \text { Closer } & & \overbrace{\text { Opened }}^{\text {Cosement }} & \text { Closed } \\ \text { Maia } & 4 & 5 & 6 & 3 \\ \text { Homarus } & 4 & 4 & \text { I } & \text { I } \\ \text { Palinurus } & 6 & 3 & 6 & 0 \\ & 5 & 6 & \text { I5 } & 5\end{array}$

The large number of fibres obtained from Palinurus resulted from (I) individual fibres being relatively larger and thus more readily prepared, and (2) there being a larger number of nerve cells in the PD-organ than in the other genera. The number of movement fibres for the opposite directions seems about equal in all genera. These counts do not show that here, as in all other joint organs, the number of position fibres is larger than that of the movement fibres. This is due to the smaller size of the position fibres, which makes them harder to prepare, and explains why in the preparation of Maia presented only a single one for each class was found, since in Maia the nerve fibres are smaller than in other genera used.

By staining the organs with methylene blue, it was observed that the elastic strand, which is so distinct in Carcinus, is a much more diffuse structure, and that the cell bodies are spread farther apart. This feature and the fact that the nerve bundle close to the organ is enveloped in a strong sheath have prevented splitting of subbundles, which showed in Carcinus the localization of the different reaction types. Since in all organs the larger cells are located at the proximal end and the signals of movement fibres are regularly larger than those of position fibres it is likely that a similar distribution is present in all organs.

\section{The proprioceptor organs in the carpo-propodite joint}

In this joint at least two separate organs are present in all genera investigated. Information about their occurrence has been given by Alexandrowicz (I958). ${ }^{1}$

\footnotetext{
${ }^{1}$ Alexandrowicz (personal communication) has suggested that receptors belonging to this system might be designated by the first letters of the segments between which they are situated, viz. PD - the organ between the propodite and the dactylopodite, $\mathrm{CP}$ - that between the carpopodite and the propodite, etc. When two organs differing somewhat in their structure are present near to one another, as at the mero-carpopodite and carpo-propodite joints, they may be distinguished by numerals, viz. MC I, MC 2 and $\mathrm{CP}$ I, $\mathrm{CP}_{2}$ respectively.
} 
In Carcinus the organs were readily found, at first by tracing back to their origin the bundles in which proprioceptive impulses were present. CP I (Fig. I A) runs from the tendon of the bender muscle (productor propoditis) to the joint membrane. $\mathrm{CP}_{2}$ (Fig. I B) originates on the tendon of the stretcher muscle (reductor propoditis) and bridges the joint, being attached to the inner wall of the propodite. In Carcinus a clear elastic strand is present in both; the one of $\mathrm{CP} 2$ resembles that of the PD organ closely, but the strand of $\mathrm{CP} \mathrm{I}$ is broader and flatter and its origin on the bender tendon less distinct.
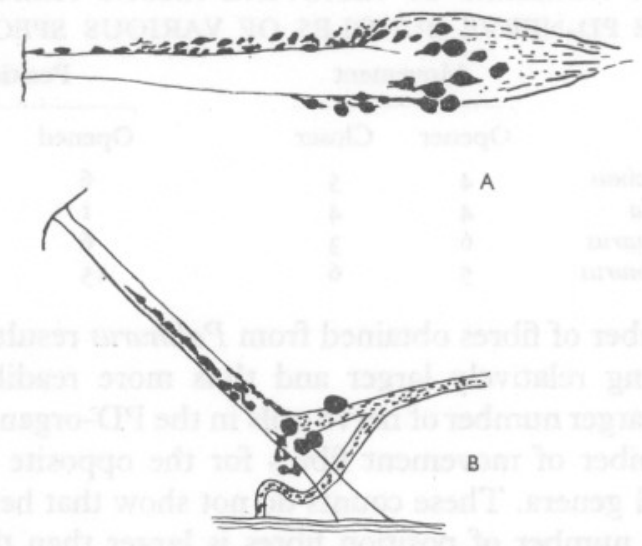

Fig. I. Carcinus maenas. A, Carpo-propodite sense organ (CP I), showing the arrangement of the nerve cells around the rather broad and proximally indefinite elastic strand. On the right the large cells of the proximal part, along the upper (outer) side the more peripheral continuation of small cells. After microphotograph of Rongalit methylene-blue stained preparation. $\mathrm{B}, \mathrm{CP} 2$ organ. Note the distinct elastic strand and the large nerve cells at the proximal end, which are in contrast to the peripheral smaller cells, loosely attached. Separate nerve branch disappears on the tendon of the stretcher muscle.

$\mathrm{CP}$ I has many more nerve cells than $\mathrm{CP} 2$ and they are clearly arranged in two main rows, one on each side of the strand. The outer row continues farther peripherally than the inner, and in both there is a rather regular decrease in cell size towards the peripheral end. In CP 2 of Carcinus a remarkable feature is the location of the large cells at the proximal end, which are here only loosely connected to the elastic strand and sometimes a considerable distance from it.

In Carcinus, as in all other genera but Palinurus, the two nerve bundles from the two organs run well separated in the main nerve trunk in the meropodite. In Palinurus they often appeared to run close together, which prevented assigning them to each organ without subsequent tracing. In Homarus and Eupagurus, where a thicker and thinner nerve bundle are present, both $\mathrm{CP}$ bundles are located in the thicker nerve.

In all genera fibres reacting to unidirectional movement as a stimulus have been found in the nerve bundles of both CP I and CP 2. Regularly 
several fibres differing in threshold but all indicating the same direction of movement are present in each bundle. Fig. $2 \mathrm{~A}$ illustrates a rather sensitive movement fibre of Homarus. The most sensitive movement fibres are not suitable for recording since very slight changes in speed will greatly influence the discharges. In Fig. 2 B medium sensitive movement fibres from Maia are shown, one for each direction, whereas insensitive fibres are represented by one from Eupagurus in Fig. $2 \mathrm{C}$.

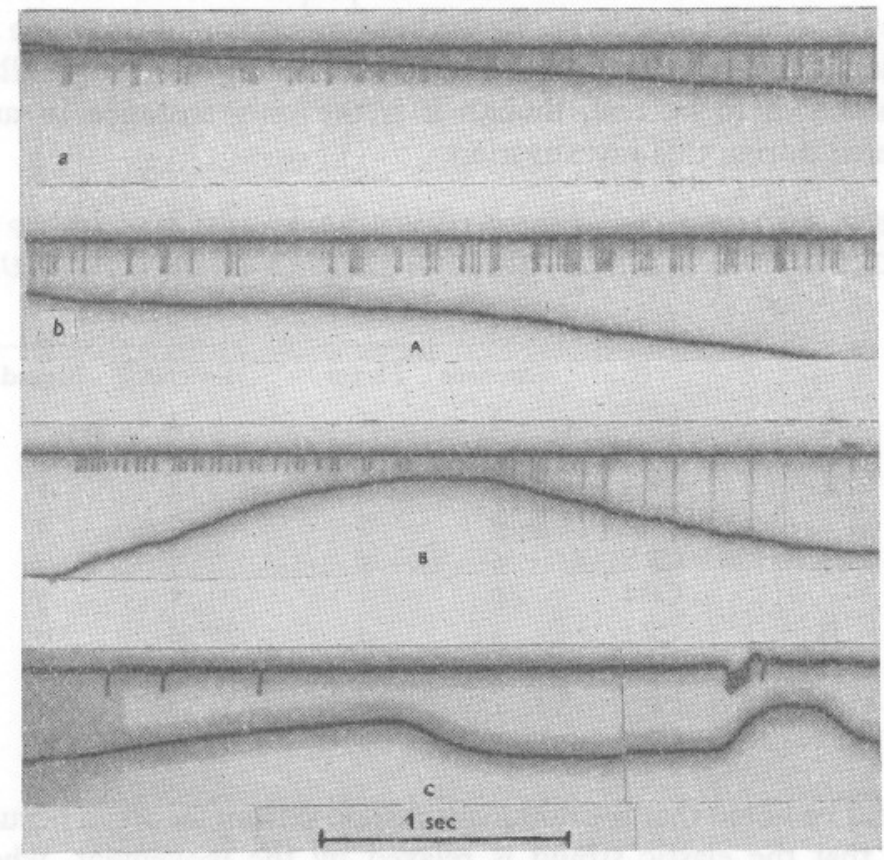

Fig. 2. A, response of sensitive flexor movement receptor from CP I of Homarus. a, start of the movement from complete extension; $b$, continuation to almost complete flexion. The irregularities in the discharge rate are mainly caused by those of the movement. $B$, a medium sensitive extensor and a flexor movement fibre from CP I of Maia, the first with the smaller signal discharging during a complete extension of the propodite, the second during flexion. $\mathrm{c}$, insensitive extension movement fibre from CP I of Eupagurus bernhardus. Two complete extensor and flexor movements, the second faster. Note the limited number of impulses this fibre can maximally produce. Time-scale I sec. during one extension.

Besides movement fibres, position fibres are invariably present in both $\mathrm{CP}$ nerve bundles. These showed no properties other than those discussed for the PD-organ of Carcinus (Wiersma \& Boettiger, 1958). Types which reacted only on position changes and types in which the frequency increased temporarily above that maintained during displacement were both noted.

In contrast to the $\mathrm{PD}$-organ the $\mathrm{CP}$-organs do not always respond to both movement directions. CP I resembles PD in this respect more than CP 2 . 
The latter may completely fail to respond to either movement or position in one direction, whereas CP I usually shows only a pronounced preference for one side. The CP-organs of Carcinus have been more closely studied in this respect but the phenomenon was present in all genera studied.

The dominant responses are extension for CP I, flexion for CP 2. Large specimens invariably showed this preference for one direction, whereas in younger ones it was less pronounced though present. Counts made of the number of active fibres which could be prepared from the two bundles illustrate this feature (Table 2). In the last preparation of this table a reversal of the dominance appears, and subsequent tracing of the bundles to the organ showed it to be real, though it is the only instance in any genus encountered during this investigation.

TABLE 2. NUMBER OF ACTIVE UNITS PREPARED IN THE CP 1 AND CP 2 NERVE BUNDLES IN FIVE LEGS (A-E) OF CARCINUS

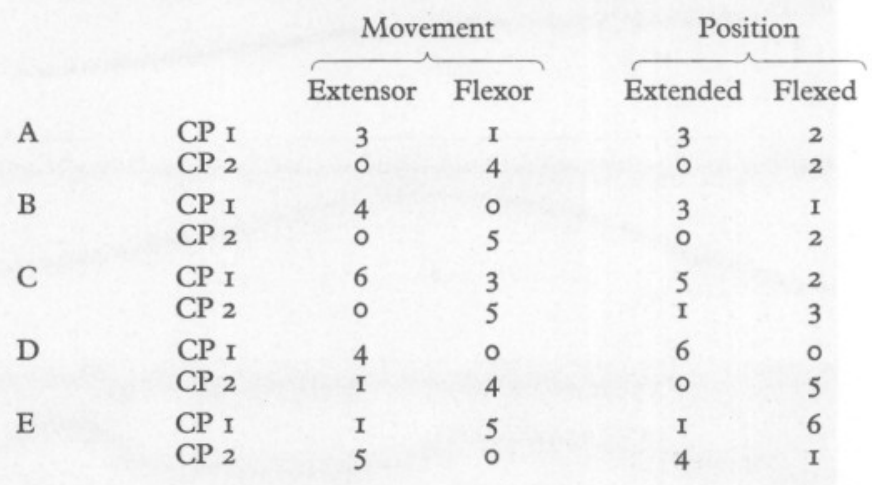

It should be noted that the dominant response from an organ occurs during the time that the elastic strand is relaxed by the movement, whereas the opposite might have been expected. That there was actual relaxation has been confirmed by studying the changes of exposed organs during the movements.

In several preparations, especially from Palinurus, fibres with a reaction to both movement and position were found. They did not occur in every preparation and were always accompanied by more conventional fibres. Sometimes there would be several of them in one bundle. Fig. $3 \mathrm{~A}$ shows a type rather common in Palinurus, whereas Fig. 3 B shows a rarer one for Homarus. As will be discussed, they may represent more or less abnormal sense cells. In Eupagurus a single fibre was found which reacted equally well to movement in either direction over the full range of the possible movement arc. This remarkable fibre is the only one so far encountered for which the name 'vibration fibre' would be appropriate. 


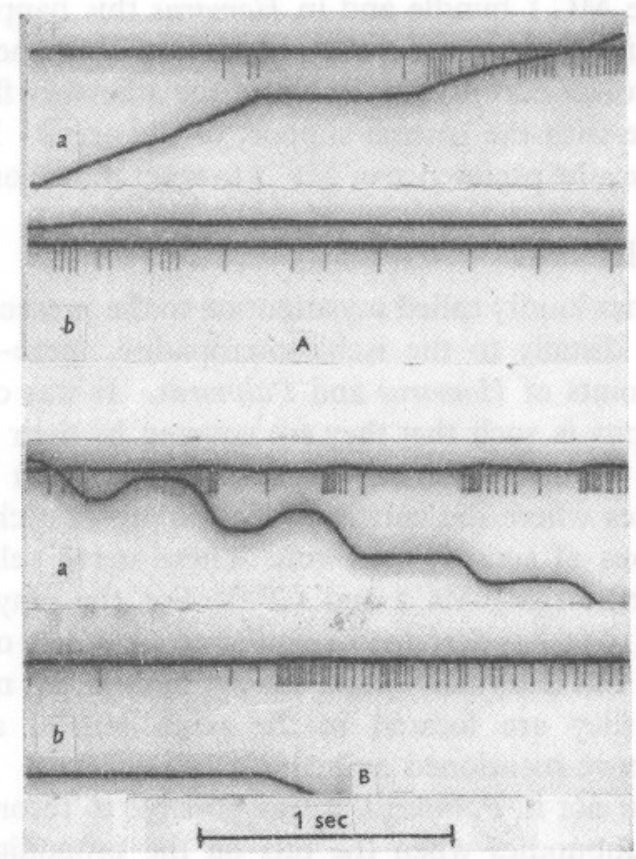

Fig. 3. A, receptor with intermediate properties from Palinurus. Insensitive to movement during the first part of the movement $\operatorname{arc}(a)$ it becomes quite sensitive for the second part and starts to respond to position though the maintained discharge rate remains low even in the extreme position $(b)$. B, receptor with intermediate properties from Homarus. Movement sensitivity present throughout the arc from the extreme extended $(a)$ to the extreme flexed position. In the latter it gives after the movement discharge a constant discharge at 20 25 impulses per sec. (b). The sections $a$ and $b$ are not continuous.

\section{$M C$-receptors}

For this joint there are again two main organs present, $\mathrm{MC}$ I which closely resembles $\mathrm{CP}_{\mathrm{I}}$ in structure and $\mathrm{MC}_{2}$ which resembles $\mathrm{CP} 2$. At least in Carcinus MC I is attached to the tendon of the accessory flexor muscle of the carpopodite (adductor carpi), and $\mathrm{MC}_{2}$ is attached close to the tendon of the main flexor muscle, and, in contrast to MC I, crosses the joint to be inserted on the inner side of the carpopodite. By exposing the nerve in the proximal part of the meropodite, leaving the distal part intact, a nerve bundle reacting to manipulation of the joint could be found for each organ. These bundles are widely separated. The bundle to MC I is easy to find as it usually leaves the main nerve trunk early in the meropodite.

In Carcinus the responses obtained from the nerve bundle of $\mathrm{MC}_{2}$, as well as fibre analysis, showed that the organ typically, but not exclusively, signals joint flexion, whereas MC I has a preponderance of extensor movement and position fibres. In some preparations of Carcinus, no signals could be 
obtained from the MC I bundle and in Homarus this happened often. The reason for such failure may be that the preparation of the nerve in the meropodite usually involves cutting the tendon of the accessory flexor muscle and hence interference with the natural support of the organ. In other genera, especially Homarus, the preference of $M \mathrm{C}_{2}$ to react to flexion was also noted.

\section{'Slit sensilla' of Homarus}

Dr Alexandrowicz kindly called my attention to the presence of a number of pits, located just distally to the ischio-meropodite, mero-carpopodite and carpo-propodite joints of Homarus and Palinurus. It was observed that the location of these pits is such that they are covered by their joint membranes when the joint is moved maximally to the side on which they occur. The 'sensilla' are places where the cuticle is pierced by fine channels to which dendritic extensions of sensory cells run. These nerve cells are associated with those of Barth-organ, $\mathrm{MC}_{2}$ and $\mathrm{CP}_{2}$. For the crayfish, Potamobius torrentium, Barth (1934) has described a similar arrangement of the innervation of 'Sinneskegel', but externally these $22-23$ sensilla do not appear to be distinct, though they are located in the same general area as the 'slit sensilla' of the above-mentioned animals.

In Homarus, but not in Palinurus, it was possible to record impulses from small prepared subbundles when the pits on the propodite were touched. The reacting nerve fibres did not run together with the $\mathrm{CP} 2$ proprioceptor fibres, but formed part of a larger bundle consisting of fibres responsive to touch of hairs on the ventral and proximal aspect of the propodite. This bundle was located near the $\mathrm{CP} 2$ bundle. By tracing the two bundles forward it was shown that the bundle of hair fibres had no connexion with the $\mathrm{CP} 2$ nerve cells. This excludes the possibility that the $\mathrm{CP} 2$ cells going to the pits are responsible for the discharges on touch. Since they might be chemoreceptors, distilled water, concentrated salt solutions and some organic material were applied to the pits, but no responses in either the $\mathrm{CP} 2$ bundle or the hair fibre bundle were obtained. It therefore remains to be shown whether or not these cells of $\mathrm{CP} 2$ are but part of the normal proprioceptor components of this organ.

From the location of the pits, it might be expected that responses would be obtained from them when they are being covered by the joint membrane. But in preparations in which touch with a brush resulted in responses, extension of the propodite at different rates, failed to do so. It is possible that under natural conditions stimulation takes place under these circumstances, as the membrane will then be more turgid through the blood pressure. 


\section{DISCUSSION}

Notwithstanding the considerable differences in the structure of the proprioceptive sense organs in different joints and different genera, they all have sense cells perceiving unidirectional movement and cells responsive to position. This strongly indicates that the as yet unknown relations between the dendrites and the elastic fibres of the strand determine the function of the sense cell. For the sense organs as a whole a development to more compactly built organs may have taken place. For instance the PD-organ of the rock lobster with its many sense cells and indistinct elastic strand may represent a more primitive state than the PD-organ of Carcinus. Physiologically a similar perfection may be seen in that fibres influenced to a great extent by both movement and position are rare in crabs and frequent in the rock lobster.

In all genera the proprioceptors of the coxal region appear to be built much more precisely than those of the peripheral joints (Alexandrowicz \& Whitear, 1957; Alexandrowicz, 1958). On the other hand, the number of sense cells in several of these organs is very small and thus a few units may transmit as detailed information as can be obtained from many of the peripheral organs. A reason for this difference may be seen in the fact that after autotomy the peripheral organs must be rebuilt. It has been observed that in regenerated legs the structure of the PD-organ can be noticeably different and that more fibres with intermediate types of responses are present. Therefore integration of many signals may be used to compensate for inaccuracy in single units.

It appears that the organs can become specialized for certain functions with ageing of the animal. This points to a functional degeneration of part of the sensory cells. How far a similar process may take place for all kinds of cells and explain the observed discrepancy between number of cell bodies and number of functionally reacting units remains to be determined.

The finding of movement sense in all groups of the Crustacea Decapoda Reptantia raises the question whether this sense is present in other Arthropoda. The available evidence strongly indicates this to be so. The responses obtained in the nerve bundles to the proprioceptive organs during the movement of joints in Limulus (see Pringle, 1956) and scorpions (Pringle, 1955) most likely originate in movement receptors. These impulses arise from large sense cells located internally, whereas smaller cells give rise to tonic discharges. According to Pringle the latter are homologous to campaniform sensilla of insects, whereas he mentions the possibility that the large cells may be homologous to the chordotonal organs. It may be that the crustacean joint organs are in a state in which these two sense organs are still combined and that the organs of the lobster represent a first stage in their divergence. It should be mentioned that in arachnoids the joint sense organs occur along the outer, upper side of the leg, whereas in Crustacea they are situated on the inner, lower side. 
I am grateful to the Director and Staff of the Plymouth Laboratory for their co-operation and for their hospitality, and especially to Dr J. S. Alexandrowicz for the help he has given me in many ways. The experiments were carried out during my tenure of a visiting Professorship at the Department of Zoology, University of Cambridge. I want to thank Prof. Sir James Gray and Staff for their confidence.

\section{SUMMARY}

Sensory fibres responding exclusively to unidirectional movement of joints are present in representative species of all four groups of Crustacea Decapoda Reptantia: Palinura, Astacura, Anomura and Brachyura.

They arise from nerve cells located in sense organs in the region of the joints, which contain additional sense cells. The axons of all these cells form bundles in the main trunk from which single unit responses were obtained by preparation of single fibres or small subbundles.

Potentially each organ contains cells sensitive to unidirectional movement for both directions and tonic position fibres for each of the sides of the mid position. Cells with different thresholds are present in each of the four classes.

In certain organs, especially those of older animals, one or two of the four types of sense cells may be lacking.

Sense cells which share some properties of movement and positionsensitive ones are always present, but truly intermediate cells are relatively scarce, more so in higher forms (crabs) than in lower ones (rock lobsters).

The functional significance of some peculiar pits in the joint regions of Homarus is studied.

The presence of movement fibres in other Arthropods is discussed and some points relating to the possible homologies of proprioceptive sense organs in the legs of Arthropods are presented.

\section{REFERENCES}

AleXandrowicz, J. S., 1958. Further observations on proprioceptors in Crustacea and a hypothesis about their function. F. mar. biol. Ass. U.K., Vol. 37, pp. 379-96.

ALEXANDROWICZ, J. S. \& WhITEAR, MARY, I957. Receptor elements in the coxal region of Decapoda Crustacea. F. mar. biol. Ass. U.K., Vol. 36, pp. 603-28.

BARTH, G., I934. Untersuchungen über Myochordotonalorgane bei dekapoden Crustaceen. Z. wiss. Zool., Bd. I45, pp. 576-624.

PRINGLE, J. W. S., I955. The function of the lyriform organs of arachnids. F. exp. Biol., Vol. 32, pp. 270-8.

- 1956. Proprioception in Limulus. F. exp. Biol., Vol. 33, pp. 658-67.

Wiersma, C. A. G. \& BoetTiger, E., I958. Unidirectional movement fibres from a proprioceptive sense organ of the crab, Carcinus maenas. F. exp. Biol., Vol. 35 (in the Press). 